\title{
Detection and recognition: Concurrent processes in perception
}

\author{
RAJA PARASURAMAN, FRANCOIS RICHER, and JACKSON BEATTY \\ University of California, Los Angeles, California 90024
}

\begin{abstract}
In two experiments, event-related brain potentials (ERPs) were recorded while subjects performed a simultaneous detection and recognition task. Ten subjects listened to pure tones in noise and reported both whether a target tone had occurred (using a four-category confidence rating scale) and whether the target was one of two (Experiment 1) or four (Experiment 2) tones differing in frequency. The amplitudes of three ERP components were found to be differentially related to detection and recognition performance. The early N100 component varied with processing related only to detection, while the late $P 300$ varied with both detection and recognition, and a later slow positive shift varied only with recognition and not with detection. While the latencies of both $\mathrm{N} 100$ and P300 increased for less confident target detections, there were no differences in the latencies of these ERP components between correctly and incorrectly identified targets. Recognition performance was above the level expected by chance even when subjects reported that no target had been presented. The results indicate that brain potential components can be used to disclose temporal features of the processing of a stimulus by the nervous system and support the view that detection and recognition are partially independent, concurrent processes in perception.
\end{abstract}

Detecting the presence of weak sensory signals in the environment forms an essential part of the perception of stimuli by the nervous system. However, detection is only one aspect of the normal perception of weak sensory events; the other is recognition or identification. In most situations, human observers must not only detect a weak signal but also identify or recognize it as a relevant or irrelevant environmental stimulus. Examples can be found in a variety of everyday and occupational perceptual tasks.

Although psychophysical models of either detection or recognition have been investigated for a number of years (Luce, 1963a; Luce \& Green, 1974; Tanner, 1956), the relationship between detection and recognition, the processes underlying simultaneous detection and recognition, and the brain events subserving these aspects of perception have only recently been studied systematically (Green \& Birdsall, 1978; Parasuraman \& Beatty, 1980; Swets, Green, Getty, \& Swets, 1978). Current areas of investigation concern questions such as whether detection and recognition are independent mechanisms, whether they are sequential, cascaded, or concurrent (parallel) temporal processes, and whether behavioral detection and recognition can be predicted from a knowledge of the underlying brain events.

We have approached these problems by examining the event-related potentials (ERPs) of the brain, which provide a unique method for assessing dy-

Supported in part by ONR Contract N00014-76-C-0616 and by NIMH Training Grant 784040-29867-5. Address reprint requests to Raja Parasuraman, Department of Psychology, University of California, Los Angeles, California 90024 .

Copyright 1982 Psychonomic Society, Inc. namic properties of brain function during cognitive processing. In simple detection tasks, both the P300 component of the ERP, a positive-going brain potential that is maximal over parietal cortex, and the N100 component, a negative potential maximal at the vertex, have been found to increase monotonically in amplitude with increases in the a posteriori likelihood ratio that a signal was presented (Hillyard, Squires, Bauer, \& Lindsay, 1971). Concomitant changes in P300 amplitude are found when the likelihood ratio is manipulated by changes in the a priori signal probability (K. C. Squires, N. K. Squires, \& Hillyard, 1975a), costs and benefits of detection (Paul \& Sutton, 1972), and confidence ratings of detection (Kerkhof, 1978; K. C. Squires, Hillyard, \& Lindsay, 1973; K. C. Squires et al., 1975b).

How are these changes in N100 and P300 amplitude to be interpreted? K. C. Squires et al. (1973) suggested that, whereas $\mathrm{N} 100$ and $\mathrm{P} 300$ are affected similarly by experimental variables in signal detection tasks, N100 appears sensitive mainly to variations in subjective signal intensity, with $\mathrm{P} 300$ being related to stimulus recognition and other decisional processes. A related view was put forward by Hillyard, Hink, Schwent, and Picton (1973), who postulated that in selective attention tasks $\mathrm{N} 100$ is associated with early stimulus (or channel) selection, whereas $P 300$ reflects detection or recognition of a specific stimulus (or target within the selected channel). If these interpretations are correct, one would predict that, in a joint detection and recognition task with more than one signal, recognition performance should be associated with P300 and not with N100, whereas detection should be related to both $\mathrm{N} 100$ and P300. The above- 
mentioned studies of $\mathrm{N} 100$ and P300 have employed only simple detection tasks. However, we recently carried out a study of ERPs in the detection and recognition of multiple-frequency acoustic signals in noise. We found that, whereas the N100 component of the ERP varied only with the detection of a signal, the P300 component varied with both detection and identification (Parasuraman \& Beatty, 1980).

Although most studies of P300 in signal detection have employed acoustic signals, weak visual or somatosensory signals that are detected also evoke large P300s (Barrett, Halliday, Halliday, \& Rudolph, 1979; Desmedt \& Debecker, 1979a, 1979b; Snyder, Hillyard, \& Galambos, 1980). The scalp distribution of P300 shows the same parietal or central focus for visual, auditory, or somatosensory signals, although visual signals evoke a P300 that peaks later and is somewhat larger than signals in other modalities (Snyder et al., 1980). These results suggest that the P300 evoked in signal detection tasks may represent an endogenous brain potential that arises from a common neural process, irrespective of the mode of stimulation. This interpretation must be tempered by the fact that the P300 may comprise several overlapping components that may respond differently to experimental manipulations (Ruchkin, Sutton, \& Stega, 1980; Simson, Vaughan, \& Ritter, 1977; N. K. Squires, K. C. Squires \& Hillyard, 1975). The contingent negative variation (CNV) shift in brain potential that precedes the stimulus "resolves" or returns to baseline following the stimulus and can contribute to changes in the characteristics of P300 (Donchin \& Smith, 1970; McCallum \& Knott, 1973). The "slow wave," a slow potential shift present in the same latency range as $\mathrm{P} 300$, can also contribute to changes in amplitude that can be erroneously attributed to P300 (N. K. Squires et al., 1975). Ruchkin et al. (1980) found that the P300 component was coincident over a portion of its latency range with both these independent components, CNV resolution and the slow wave; the components differed in scalp distribution and task effects and contributed to the changes in P300 found using a baseline-to-peak measure.

These findings indicate the importance of accounting for the influence of overlapping components on P300, if the P300 and other brain potential components are to be utilized as reliable temporal indices of information processing in the nervous system. In our previous brief report (Parasuraman \& Beatty, 1980), we did not present findings on the possible influence of overlapping components on P300 in detection and recognition. We now present a more detailed account of the findings from this study that show that, although the P300 overlaps with a slow positive shift (SPS) in potential, the previously reported $\mathrm{P} 300$ results remain robust. In addition, we found that the N100, P300, and SPS components of the ERP were differentially related to detection and recognition.
In the present study, ERPs were recorded during the detection and recognition of weak acoustic signals embedded in noise. We employed both conventional baseline-to-peak measures of ERP components and measures based on independent components extracted by multivariate analysis.

\section{METHOD}

\section{Subjects}

Ten subjects, six males and four females aged 18 to 32 years, volunteered to participate in the study. Six subjects took part in Experiment 1 and four in Experiment 2. All subjects had normal hearing and normal or corrected-to-normal vision.

\section{Detection and Recognition Task}

Subjects sat at a computer terminal and listened through Superex headphones to tones presented binaurally against continuous wideband noise ( $60 \mathrm{~dB}$ SPL). Each trial began with a $100-\mathrm{msec}$ presentation of the word "READY" on the terminal display, followed $600 \mathrm{msec}$ later, on half the trials, by a $50-\mathrm{msec}$ tone drawn equiprobably from a set of either two (Experiment 1) or four (Experiment 2) targets differing in frequency. On the remaining trials, noise alone was presented. Fifteen-hundred milliseconds after the warning signal, a response cue (the word "RESPOND" on the display) directed subjects to use a four-category confidence rating scale ( 1 , yes-sure; 2 , yes-unsure; 3 , no-unsure; 4 , no-unsure) to indicate whether they had detected a target and to depress one of either two or four recognition response keys to indicate target type. Subjects were required to make recognition judgments on every trial, irrespective of their detection judgment. The intertrial interval varied randomly from 2 to $6 \mathrm{sec}$.

The targets were pure tones generated digitally to have a zero starting and ending phase, 10-msec rise and fall times (exponentially ramped), and $30 \mathrm{msec}$ at full 12-bit amplitude. For the target set size of two, the tone frequencies were 900 and $1,400 \mathrm{~Hz}$. For the target set size of four, the tone frequencies were $600,1,100$, 1,700 , and $2,200 \mathrm{~Hz}$. The signal to noise ratios (E/No) were adjusted for each tone and individually for each subject so that detectability, as indexed by $\mathrm{d}^{\prime}$, was about 1.3 for each signal. As a first approximation, the following formula was used to equate detectability at the different frequencies: $10 \log (\mathrm{E} / \mathrm{No})=2(\mathrm{~F} / \mathrm{Fc})$ $+8 \mathrm{~dB}$ (where $\mathrm{Fc}=1,000 \mathrm{~Hz}, \mathrm{~F}=$ signal frequency). (Green, McKey, \& Licklider, 1959). Small increments or decrements in intensity were then made to adjust detectability levels for different subjects. Signal levels across subjects were within $2 \mathrm{~dB}$ of each other.

\section{Recording System}

The electroencephalogram (EEG) was recorded using nonpolarizable, silver-silver chloride electrodes placed on the subject's scalp. In Experiment 1, the electrodes were placed over midline frontal $(\mathrm{Fz})$, central $(\mathrm{Cz})$, and parietal $(\mathrm{Pz})$ cortex, as well as over left $(\mathrm{C} 3)$ and right $(\mathrm{C} 4)$ central cortex. Only the midline placements were used in Experiment 2. Linked earlobe electrodes provided the reference in both cases. The electrooculogram (EOG) was recorded from infra- and supraorbital electrodes placed about the left eye. Interelectrode impedances were kept below $5 \mathrm{k} \mathcal{Q}$. The EEG and EOG were amplified (Grass Model 8; bandpass .1 to $35 \mathrm{~Hz}$ ) and digitized at 12 bits (PDP-11/34). A 1,600-msec epoch, beginning $100 \mathrm{msec}$ before the warning stimulus and ending at the response cue, was sampled at $80 \mathrm{~Hz}$ and stored on magnetic tape.

The EEG and the EOG were monitored on-line for the presence of ocular and muscular artifacts. The rms value of the EOG signal was computed and compared with a preset individual criterion after each trial. In addition, a moving-window algorithm detected the presence of sharp transients due to eye blinks and body movement. Finally, the EEG record was inspected visually for muscle artifacts not appearing in the EOG channel. This information was 
written along with the EEG and EOG data on magnetic tape for off-line rejection of artifact-contaminated trials. A running count of the number of contaminated and acceptable trials was continuously available to the experimenter to enable him to estimate the number of experimental blocks needed to acquire a minimum of 1,000 artifact-free trials. The artifact rejection rate varied between $5 \%$ and $15 \%$ for different subjects.

\section{Procedure}

Each subject participated in from three to five sessions, each lasting about $2 \mathrm{~h}$. About $3 \mathrm{~h}$ of practice preceded any data collection. During the initial practice and training trials, the tones were presented at large signal-to-noise ratios so that each target tone could be recognized reliably. Following the practice sessions, each subject received between 1,200 and 1,300 trials in blocks of 50-80 trials each. Short rest periods were provided between blocks, during which the subjects were given feedback regarding their current level of performance. Each subject was paid an hourly wage that was remitted at the end of the last session.

\section{Data Analysis}

Analysis of detection and recognition performance. In a simple detection task with a four-category confidence rating scale, each subject's performance can be summarized with a three-point receiver operating characteristic (ROC). In a combined detection and recognition task, both simple detection and combined detection and recognition ROCs can be obtained. The first kind of ROC is a plot of the probability of correct target detections (or hits) vs. false alarms at the different response criteria separating adjacent confidence categories. The ROC is obtained in the same manner as for a "simple" detection task having a single target by treating all targets the same. The detection and recognition ROC is the ROC resulting when the "hit" response is redefined to include correct recognition as well as detection (see Green \& Birdsall, 1978; Swets \& Birdsall, 1978). The probability of a correct detection and recognition response in the joint $R O C$ is smaller than the probability of a simple detection at the same criterion; the theorem of Starr, Metz, Lusted, and Goodenough (1975) predicts the extent of the reduction to be a function of the size of the target set and the criterion level, assuming targets are orthogonal and of equal detectability. The theorem of Starr et al. shows that the probability of a correct recognition (R) and detection (D) response at a given criterion level (c), or $\mathrm{Pc}(\mathrm{D} \& \mathrm{R} / \mathrm{s})$, can be predicted from the simple detection probability $P(D / s)$, namely:

$$
\operatorname{Pc}(D \& R / s)=\underset{i}{\operatorname{Pc}(D / s)}-\frac{(M-1)}{M} \int_{0}^{c_{i} 1-\operatorname{Pc}(D / s)} \frac{1-\operatorname{Pc}(D / n)}{1 P c}(D / n),
$$

where $M$ is the target set size and $P(D / n)$ is the false-alarm probability at the criterion level $c$. The integral was estimated, using an area measure:

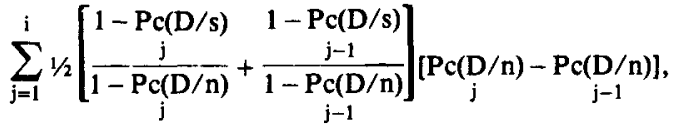

$$
\begin{aligned}
& \text { where } \frac{1-P c(D / s)}{1-P c(D / n)} \rightarrow 1 \text { as } P(D / n) \rightarrow 0 \text {. }
\end{aligned}
$$

The theorem of Starr et al. (1975) was used to assess the degree of dependence between detection and recognition by examining whether the joint detection and recognition $R O C$ could be predicted from the simple detection ROC.

ROC analysis was also used to evaluate detection performance. The simple detection ROCs were fitted, using an iterative maximumlikelihood procedure (Grey \& Morgan, 1972). The fitted solution was then used to derive the detectability index $\mathrm{d}_{\mathrm{a}}$ (Simpson \& Fitter, 1973) for each target.

Analysis of ERPs. ERPs were analyzed in three phases. In the first phase, conventional ensemble averages were computed and examined for waveshape, scalp distribution, and experimental effects. ERPs were averaged separately for each combination of stimulus and response types over the 1,600-msec recording epoch. A small negative shift in baseline potential, the contingent negative variation (CNV), was observed in the interval between the warning stimulus and the target stimulus. CNV amplitude was measured as the maximum negative deflection in this interval relative to a 100 -msec prewarning stimulus baseline. In the posttarget epoch, a prominent $\mathrm{P} 300$ component and a smaller N100 component were the two major peaks observed. The amplitudes of these components were measured relative to a 100 -msec prestimulus baseline. The measurement intervals for the peaks of N100 and P300 were 80-170 and $250-500 \mathrm{msec}$, respectively. The target trial EEG epochs were further analyzed to yield ERPs for each target-response outcome. ERPs were obtained for each cell of the "confusion matrix" between target type and identification response; there were 4 cells for the 2 targets in Experiment 1 and 16 cells for the 4 targets in Experiment 2. Because of the small number of certain targetresponse outcomes, only ERPs collapsed across ratings 1 and 2 were analyzed.

In the second phase, adaptive filtering techniques were utilized in the analysis of single-trial target ERPs. This analysis, which was restricted to a 1,000 -msec epoch beginning $100 \mathrm{msec}$ before the target stimulus, was carried out to validate the results obtained using averaged ERP analysis, as well as to predict recognition on a trial-by-trial basis using single-trial estimates of P300 amplitude. The single-trial sampled EEG epochs were prefiltered with a minimum mean-square error digital filter. Filter coefficients were chosen individually for each subject to remove high-frequency EEG components and EEG components uncorrelated with the ERP. A peak selection and detection routine modified from one developed by McGillem and Aunon (1977) was then used over a restricted search interval (200-900 msec poststimulus) to obtain an estimate of the mean amplitude, latency, and latency variability of $\mathrm{P} 300$ across single trials.

In the third phase, the target ERPs were subjected to a principal components analysis (PCA) (Donchin and Heffley, Note 1) using the BMDP4M program (Dixon, 1977). As for the single-trial procedure, the epoch for analysis was $1,000 \mathrm{msec}$ long, beginning $100 \mathrm{msec}$ before the presentation of the target stimulus, and subjects were included from both Experiments 1 and 2 (ERP data from electrode sites $\mathrm{C} 3$ and $\mathrm{C} 4$ in Experiment 1 were not utilized). A total of 240 ERP cases (10 subjects $\times 2$ recognition levels $\times 4$ detection levels $\times 3$ electrode sites) were employed in the analysis. Principal components were extracted for both the covariance and the cross-product matrices formed from the sample points. A number of PCA solutions were examined to investigate the robustness of the obtained components and, in the final analysis, seven components were extracted and rotated by the Varimax criterion method.

\section{RESULTS}

\section{Behavioral Detection and Recognition}

Figure 1 plots the simple detection and joint detection and recognition ROCs for each of the 10 subjects in Experiments 1 and 2. It can be seen that the recognition ROCs are displaced downward relative to the detection ROCs. Note also that, although the detection ROC is defined by three points, the recognition ROC is characterized by four data points. This is because subjects were asked to make identification responses even when they reported being sure that no signal was presented.

The detection ROCs in Figure 1 were fitted by eye; for the purposes of analysis of detection performance, each ROC was fitted, using a maximum- 
likelihood procedure and the detectability index $d_{a}$ was derived. The mean values of $d_{a}$ for the $900-$ and $1,400-\mathrm{Hz}$ targets in Experiment 1 were 1.05 and 1.15, respectively; these values did not differ significantly. Although in Experiment 2 there was a tendency for the 1,100- and $1,700-\mathrm{Hz}$ (inner frequency) targets to be less detectable (1.13 and 1.00 , respectively) than the $900-$ and $2,200-\mathrm{Hz}$ (outer frequency) targets (1.18 and 1.17 , respectively), these differences were not significant.

Given targets that are equally detectable and orthogonal, the theorem of Starr et al. (1975) can be used to predict the joint detection and recognition ROC. Table 1 gives the mean values of the obtained and predicted joint detection and recognition probabilities at each confidence rating. As Table 1 indicates, the obtained and predicted values were monotonically related, but the theorem consistently overestimated observer performance.

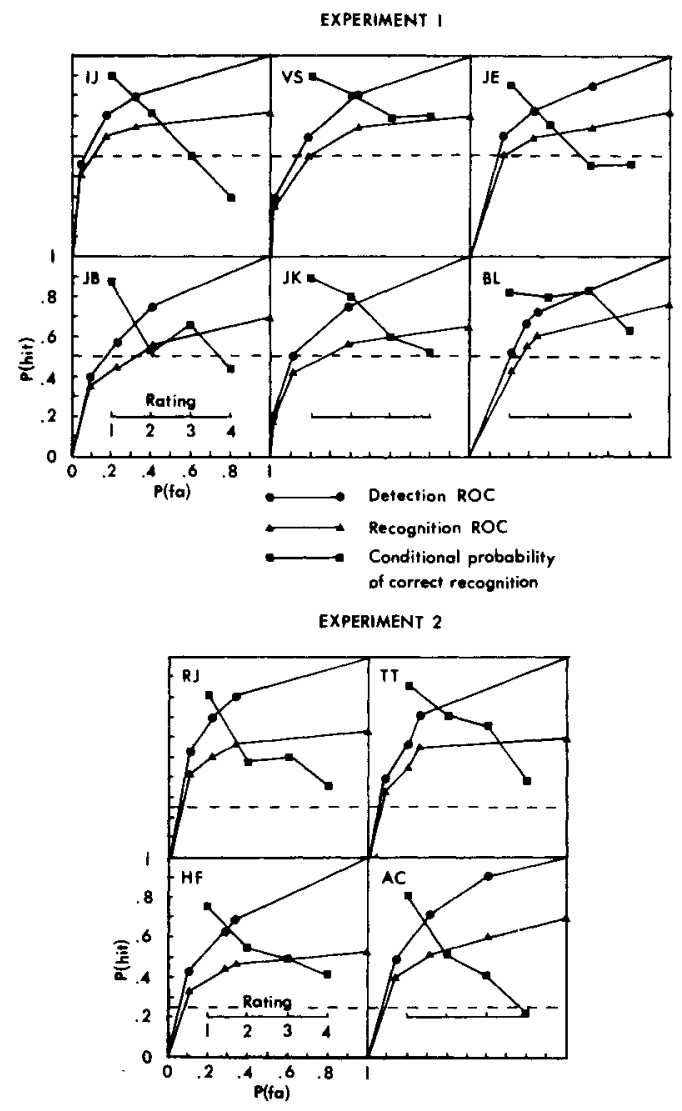

Figure 1. Individual detection and recognition receiver operating characteristics (ROCs) for the 10 subjects in Experiments 1 and 2. In the recognition $\mathrm{ROC}$, the "hit" response is redefined to include both correct detection and recognition of the target. Also shown are the conditional probabilities of correct recognition given each confidence rating (1, yes-sure; 2 , yes-unsure; 3 , no-unsure; 4 , no-sure); the dotted lines show the recognition probability to be expected by chance alone ( .50 for the two targets in Experiment 1 and $\mathbf{2 5}$ for the four targets in Experiment 2).
Table 1

Mean Values of the Joint Probability of Detection and Recognition, P(D\&R), at Each Confidence Rating

\begin{tabular}{|c|c|c|c|c|c|c|}
\hline \multirow[b]{3}{*}{ Rating } & \multicolumn{3}{|c|}{ Experiment 1} & \multicolumn{3}{|c|}{ Experiment 2} \\
\hline & \multicolumn{2}{|c|}{$P(D \& R)$} & \multirow[b]{2}{*}{$\mathrm{P}(\mathrm{R})$} & \multicolumn{2}{|c|}{$P(D \& R)$} & \multirow[b]{2}{*}{$P(R)$} \\
\hline & $\mathrm{O}$ & $\mathbf{P}$ & & $\mathrm{O}$ & $\mathbf{P}$ & \\
\hline 1 & .35 & .37 & .87 & .37 & .39 & .81 \\
\hline 2 & .16 & .17 & .74 & .11 & .14 & .56 \\
\hline 3 & .10 & .13 & .62 & .07 & .10 & .52 \\
\hline 4 & .12 & .17 & .42 & .08 & .11 & .35 \\
\hline
\end{tabular}

Note $-P(D \& R)$ values are based on 600-650 target trials/subject. Confidence ratings: $1=y e s-$ sure; $2=y e s-$ unsure; $3=$ nounsure; $4=$ no-sure. The experimentally obtained (O) values are shown along with the values predicted $(P)$ from detection performance using Starr et al.'s (1975) theorem. The mean values of the probability of a correct target recognition given a detection, $P(R)$, are also given. The chance level of correct recognition was .50 in Experiment 1 (two targets) and .25 in Experiment 2 (four targets).

Table 1 also gives the mean values of the conditional probability of recognition given detection for each confidence rating. Individual values are plotted in Figure 1. As expected, recognition probability was highest at the most confident detection rating 1 , and decreased across ratings 2,3 , and 4 . More importantly, recognition probability was above the level of chance even when subjects made doubtful targetabsent reports (rating 3). (See Figure 1 and Table 1.) This was confirmed in 9 of the 10 subjects, using a $\chi^{2}$ test corrected for small sample size $\left[\chi^{2}(1)=4.9\right.$, $\mathrm{p}<.05]$. For rating 4, however, recognition probability was not significantly higher than chance level; recognition performance was above chance for six subjects and at or below chance level for four subjects.

\section{Averaged ERP Analysis}

CNV in detection and recognition. Figures 2 and 3 display average ERPs for correctly and incorrectly identified targets at each confidence rating of detection for two subjects in Experiments 1 and 2. The ERPs are characterized by a small CNV in the warning interval and by a prominent P300 and smaller N100 in the posttarget epoch.

Table 2 gives the mean values of the amplitude of the CNV for each condition in Experiments 1 and 2. A three-way analysis of variance (correctness of recognition $\times$ detection rating $x$ electrode site) showed that there were no significant differences in CNV amplitude with confidence rating of detection or with correctness of recognition. The only significant effect on CNV amplitude was that due to electrode site [Experiment 1: $F(4,20)=41.9, p<.001 ;$ Experiment 2: $F(2,6)=73.5, \mathrm{p}<.001]$; in both cases, the $\mathrm{CNV}$ was more negative at $\mathrm{Fz}$ and $\mathrm{Cz}$ than at $\mathrm{Pz}$.

N100 and P300 in detection and recognition. Figure 4 shows the target ERPs (for a 1,000-msec epoch 


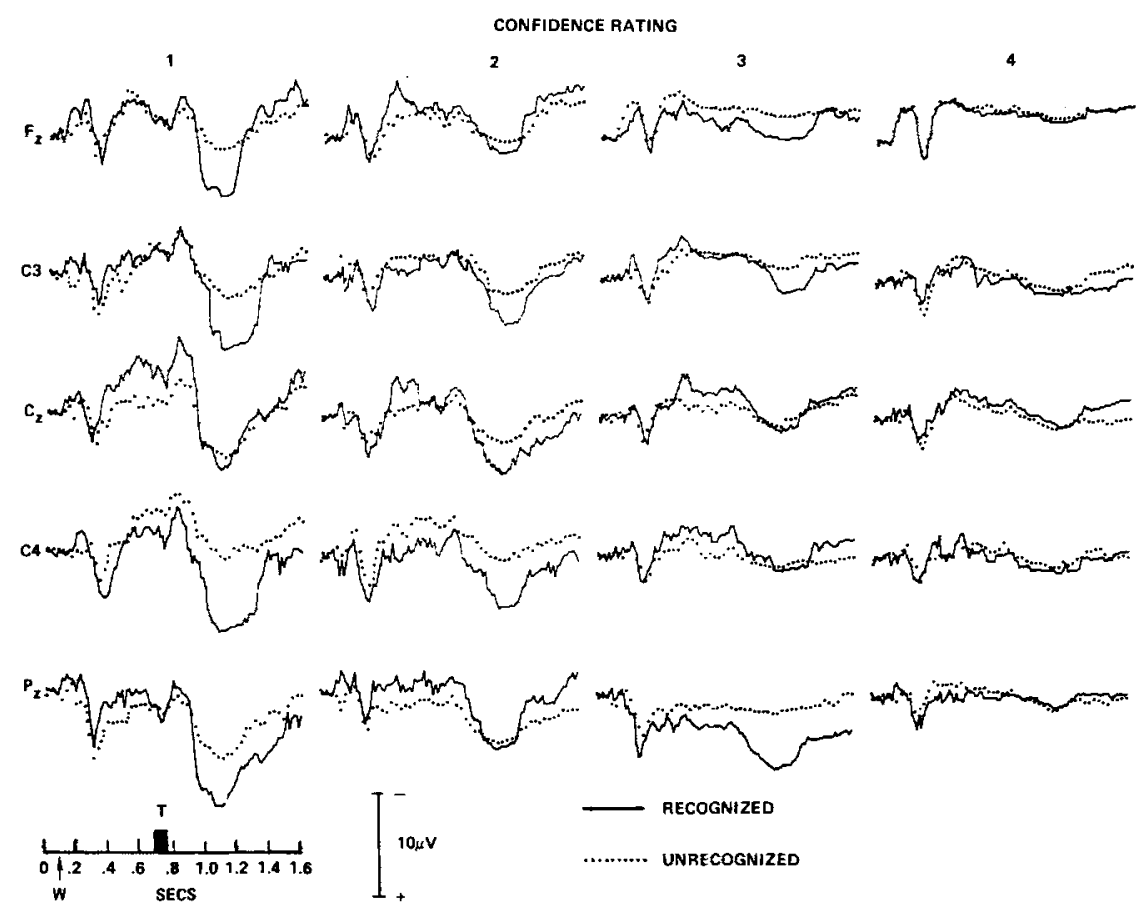

Figure 2. ERPs for one subject from Experiment 1, averaged separately for correctly and incorrectly recognized targets and for each confidence rating, at each of the five electrode sites. The times of presentation of the target (T) and of the warning stimulus (W) are also shown.

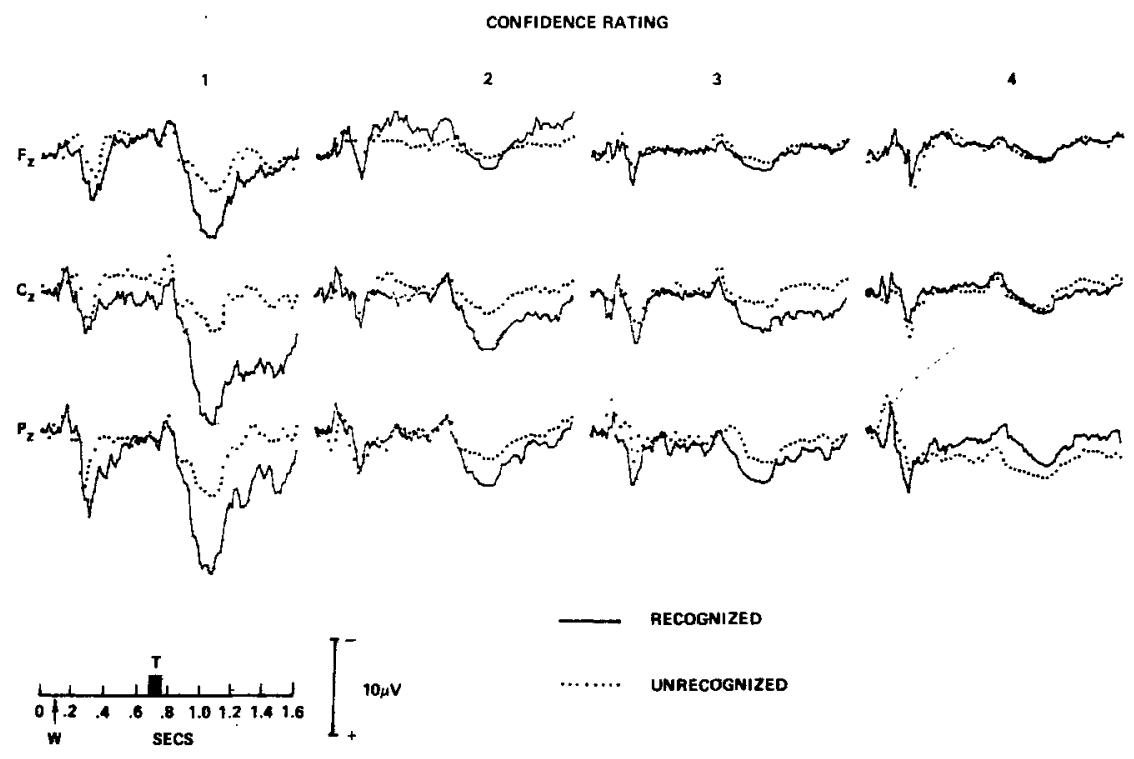

Figure 3. Target ERPs at each of the three electrode sites for one subject in Experiment 2. 
Table 2

Mean Values of CNV Amplitude (in Microvolts) for Recognized (R) and Unrecognized (U) Targets at Each Detection Rating

\begin{tabular}{rrrrrr}
\hline & & \multicolumn{4}{c}{ Confidence Rating } \\
\cline { 3 - 6 } & & & \multicolumn{4}{c}{2} & 3 & \multicolumn{1}{c}{4} \\
\hline & & & \multicolumn{4}{c}{ Experiment 1 } \\
$\mathrm{Fz}$ & $\mathrm{R}$ & -4.30 & -5.07 & -4.80 & -4.83 \\
& $\mathrm{U}$ & -4.67 & -4.87 & -5.60 & -5.63 \\
$\mathrm{C} 3$ & $\mathrm{R}$ & -1.53 & -1.33 & -1.47 & -1.33 \\
& $\mathrm{U}$ & -1.40 & -1.67 & -1.33 & -1.20 \\
$\mathrm{Cz}$ & $\mathrm{R}$ & -2.40 & -1.97 & -2.13 & -2.13 \\
& $\mathrm{U}$ & -2.30 & -2.20 & -2.60 & -.93 \\
$\mathrm{C} 4$ & $\mathrm{R}$ & -.60 & -1.47 & -2.20 & -.57 \\
& $\mathrm{U}$ & -2.07 & -1.87 & -.93 & -.47 \\
$\mathrm{Pz}$ & $\mathrm{R}$ & .63 & .60 & .60 & 1.63 \\
& $\mathrm{U}$ & .97 & 1.47 & 1.53 & .87 \\
& & & Experiment 2 & \\
$\mathrm{Fz}$ & $\mathrm{R}$ & -3.05 & -2.90 & -1.70 & -2.35 \\
& $\mathrm{U}$ & -2.90 & -2.70 & -1.15 & -2.90 \\
$\mathrm{Cz}$ & $\mathrm{R}$ & -1.70 & -.95 & -.75 & -1.20 \\
& $\mathrm{U}$ & -1.05 & -1.25 & -1.60 & -1.70 \\
$\mathrm{Pz}$ & $\mathrm{R}$ & .30 & .25 & .40 & .55 \\
& $\mathrm{U}$ & .50 & .55 & .00 & .20 \\
\hline
\end{tabular}

Note-Values are given for each electrode site.

beginning $100 \mathrm{msec}$ before the target stimulus) averaged over subjects and electrode sites for both Experiments 1 and 2 . The ERPs, which were averaged separately according to confidence rating of detection and correctness of recognition, disclose effects for both the N100 and P300 components. Table 3 gives the mean values of P300 amplitude for each condition in both Experiments 1 and 2.
Three-way analysis of variance of $\mathrm{P} 300$ amplitude gave significant effects for correctness of recognition [Experiment 1: $\mathrm{F}(1,5)=49.8, \mathrm{p}<.001$; Experiment 2: $F(1,3)=49.4, p<.001]$ and for the interaction between recognition and confidence rating [Experiment 1: $\mathrm{F}(3,15)=16.5, \mathrm{p}<.001 ; \quad$ Experiment 2: $F(3,9)=21.9, p<.001]$. P300 amplitude was significantly larger for correctly recognized targets than for incorrectly identified targets for confidence ratings 1,2 , and 3 only [simple effects, Experiment 1: $F(1,5)$ $>19.1, \mathrm{p}<.05$; Experiment 2: $\mathrm{F}(1,3)>34.1, \mathrm{p}<.01$, for each case] and not at rating 4 . In addition, P300 amplitude decreased significantly across the detection ratings 1 to 4 [Experiment $1: F(3,5)=59.4$, p < .001 ; Experiment 2: $\mathrm{F}(3,3)=39.8, \mathrm{p}<.001$ ]. There was a significant effect of electrode site on P300 amplitude in both Experiment $1[\mathrm{~F}(4,20)=17.8, \mathrm{p}<.001]$ and Experiment $2[\mathrm{~F}(2,6)=13.2, \mathrm{p}<.01]$; in both cases, P300 was smaller at $\mathrm{Fz}$ than at $\mathrm{Cz}$ or $\mathrm{Pz}$.

Table 4 gives the mean values of N100 amplitude for each experimental condition. N100 amplitude differed significantly across electrode sites in both Experiment $1[\mathrm{~F}(4,20)=34.9, \mathrm{p}<.001]$ and Experiment $2[\mathrm{~F}(2,6)=46.9, \mathrm{p}<.01]$; in both cases, N100 was largest at the vertex $(\mathrm{Cz})$ than at the other electrode sites. N100 decreased significantly in amplitude across confidence ratings 1 to 4 [Experiment 1: $F(3,5)$ $=14.3, \mathrm{p}<.01$; Experiment 2: $\mathrm{F}(3,3)=5.8, \mathrm{p}<.10]$. However, N100 amplitude did not differ significantly between correctly and incorrectly identified targets. Thus, while P300 amplitude was affected by both detection and recognition, only detection affected N100 amplitude (see Figures 2, 3, and 4).

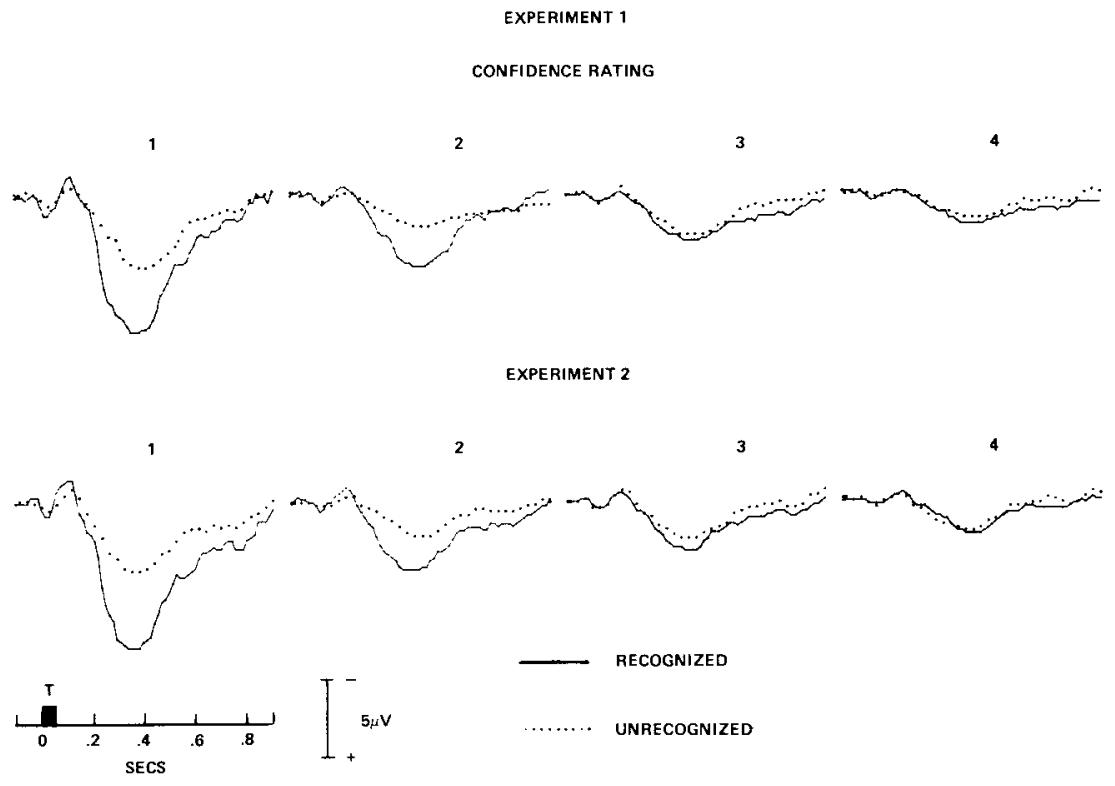

Figure 4. Grand average ERPs (averaged over subjects and electrode sites) for correctly and incorrectly recognized targets and for each confidence rating (1, yes-sure; 2 , yes-unsure; 3 , no-unsure; 4, no-sure). 
Table 3

Mean Values of P300 Amplitude (in Microvolts) for Recognized (R) and Unrecognized (U) Targets at Each Detection Rating

\begin{tabular}{|c|c|c|c|c|c|}
\hline & & \multicolumn{4}{|c|}{ Confidence Rating } \\
\hline & & 1 & 2 & 3 & 4 \\
\hline & & \multicolumn{4}{|c|}{ Experiment 1} \\
\hline $\mathrm{Fz}$ & $\begin{array}{l}\mathbf{R} \\
\mathbf{U}\end{array}$ & $\begin{array}{l}7.26 \\
3.64\end{array}$ & $\begin{array}{l}3.46 \\
1.76\end{array}$ & $\begin{array}{r}1.74 \\
.76\end{array}$ & $\begin{array}{r}1.40 \\
.94\end{array}$ \\
\hline C3 & $\begin{array}{l}\mathrm{R} \\
\mathrm{U}\end{array}$ & $\begin{array}{l}8.46 \\
5.10\end{array}$ & $\begin{array}{l}4.34 \\
2.46\end{array}$ & $\begin{array}{l}2.56 \\
1.40\end{array}$ & $\begin{array}{l}1.86 \\
1.30\end{array}$ \\
\hline $\mathrm{Cz}$ & $\begin{array}{l}\mathrm{R} \\
\mathrm{U}\end{array}$ & $\begin{array}{l}9.54 \\
6.20\end{array}$ & $\begin{array}{l}5.50 \\
3.16\end{array}$ & $\begin{array}{l}3.54 \\
2.24\end{array}$ & $\begin{array}{l}2.84 \\
2.14\end{array}$ \\
\hline $\mathrm{C} 4$ & $\begin{array}{l}\mathbf{R} \\
\mathbf{U}\end{array}$ & $\begin{array}{l}8.66 \\
5.24\end{array}$ & $\begin{array}{l}4.64 \\
2.64\end{array}$ & $\begin{array}{l}2.00 \\
1.54\end{array}$ & $\begin{array}{l}1.50 \\
1.14\end{array}$ \\
\hline \multirow[t]{2}{*}{$\mathrm{Pz}$} & $\begin{array}{l}\mathbf{R} \\
\mathbf{U}\end{array}$ & $\begin{array}{l}9.70 \\
6.26\end{array}$ & $\begin{array}{l}5.36 \\
3.30\end{array}$ & $\begin{array}{l}3.96 \\
1.26\end{array}$ & $\begin{array}{l}2.74 \\
2.24\end{array}$ \\
\hline & & \multicolumn{4}{|c|}{ Experiment 2} \\
\hline $\mathrm{Fz}$ & $\begin{array}{l}\mathbf{R} \\
\mathbf{U}\end{array}$ & $\begin{array}{l}8.30 \\
4.90\end{array}$ & $\begin{array}{l}4.00 \\
1.70\end{array}$ & $\begin{array}{l}2.26 \\
1.60\end{array}$ & $\begin{array}{l}1.70 \\
1.60\end{array}$ \\
\hline $\mathrm{Cz}$ & $\begin{array}{l}\mathbf{R} \\
\mathbf{U}\end{array}$ & $\begin{array}{r}10.46 \\
4.96\end{array}$ & $\begin{array}{l}5.00 \\
2.56\end{array}$ & $\begin{array}{l}3.80 \\
2.26\end{array}$ & $\begin{array}{l}2.76 \\
2.26\end{array}$ \\
\hline $\mathrm{Pz}$ & $\begin{array}{l}\mathbf{R} \\
\mathbf{U}\end{array}$ & $\begin{array}{r}10.60 \\
5.70\end{array}$ & $\begin{array}{l}5.36 \\
3.26\end{array}$ & $\begin{array}{l}3.86 \\
2.50\end{array}$ & $\begin{array}{l}2.16 \\
2.36\end{array}$ \\
\hline
\end{tabular}

Note-Values are given for each electrode site.

The peak latencies of the N100 and P300 components were affected only by detection rating and not by correctness of recognition. The latency of both N100 [Experiment 1: F(3,5)=23.8, $\mathrm{p}<.01$; Experiment 2: $F(3,3)=20.1, \mathrm{p}<.025]$ and $P 300$ [Experiment 1: $F(3,5)=29.8, \mathrm{p}<.01$; Experiment $2: F(3,3)$ $=18.2, \mathrm{p}<.025]$ increased across detection ratings 1 to 4 .

Table 4

Mean Values of N100 Amplitude (in Microvolts) for Recognized (R) and Unrecognized (U) Targets for Each Detection Rating

\begin{tabular}{|c|c|c|c|c|c|}
\hline & & \multicolumn{4}{|c|}{ Confidence Rating } \\
\hline & & 1 & 2 & 3 & 4 \\
\hline & & \multicolumn{4}{|c|}{ Experiment 1} \\
\hline $\mathrm{Fz}$ & $\begin{array}{l}\mathbf{R} \\
\mathbf{U}\end{array}$ & $\begin{array}{r}1.26 \\
.96\end{array}$ & $\begin{array}{l}.50 \\
.36\end{array}$ & $\begin{array}{l}.30 \\
.34\end{array}$ & $\begin{array}{l}.34 \\
.34\end{array}$ \\
\hline $\mathrm{C} 3$ & $\begin{array}{l}\mathbf{R} \\
\mathbf{U}\end{array}$ & $\begin{array}{l}1.46 \\
1.36\end{array}$ & $\begin{array}{l}.74 \\
.66\end{array}$ & $\begin{array}{l}.54 \\
.44\end{array}$ & $\begin{array}{l}.36 \\
.26\end{array}$ \\
\hline $\mathrm{Cz}$ & $\begin{array}{l}R \\
U\end{array}$ & $\begin{array}{l}1.96 \\
1.94\end{array}$ & $\begin{array}{l}.96 \\
.86\end{array}$ & $\begin{array}{l}.56 \\
.64\end{array}$ & $\begin{array}{l}.44 \\
.34\end{array}$ \\
\hline $\mathrm{C} 4$ & $\begin{array}{l}\mathbf{R} \\
\mathbf{U}\end{array}$ & $\begin{array}{l}1.60 \\
1.26\end{array}$ & $\begin{array}{l}.96 \\
.74\end{array}$ & $\begin{array}{l}.50 \\
.64\end{array}$ & $\begin{array}{l}.30 \\
.30\end{array}$ \\
\hline \multirow[t]{2}{*}{$\mathbf{P z}$} & $\begin{array}{l}\mathbf{R} \\
\mathbf{U}\end{array}$ & $\begin{array}{r}1.16 \\
.96\end{array}$ & $\begin{array}{l}.54 \\
.40\end{array}$ & $\begin{array}{l}.34 \\
.46\end{array}$ & $\begin{array}{l}.34 \\
.24\end{array}$ \\
\hline & & \multicolumn{4}{|c|}{ Experiment 2} \\
\hline $\mathrm{Fz}$ & $\begin{array}{l}\mathbf{R} \\
\mathbf{U}\end{array}$ & $\begin{array}{l}1.56 \\
1.20\end{array}$ & $\begin{array}{l}.74 \\
.56\end{array}$ & $\begin{array}{l}.56 \\
.66\end{array}$ & $\begin{array}{l}.60 \\
.36\end{array}$ \\
\hline $\mathrm{Cz}$ & $\begin{array}{l}R \\
U\end{array}$ & $\begin{array}{l}2.20 \\
1.74\end{array}$ & $\begin{array}{l}1.30 \\
1.10\end{array}$ & $\begin{array}{r}.96 \\
1.10\end{array}$ & $\begin{array}{l}.80 \\
.86\end{array}$ \\
\hline $\mathrm{Pz}$ & $\begin{array}{l}\mathbf{R} \\
\mathrm{U}\end{array}$ & $\begin{array}{l}1.90 \\
1.50\end{array}$ & $\begin{array}{l}1.06 \\
1.06\end{array}$ & $\begin{array}{l}.74 \\
.80 \\
\end{array}$ & $\begin{array}{r}.80 \\
.66 \\
\end{array}$ \\
\hline
\end{tabular}

Note-Values are given for each electrode site.
P300 amplitude and the recognition confusion matrix. Table 5 gives the mean values of P300 amplitude for ERPs averaged separately for each cell of the confusion matrix between target type and recognition response in Experiments 1 and 2. P300 amplitude was greater for cells along the diagonal (correct target identifications) than for off-diagonal cells (incorrect identifications), confirming the effect of recognition obtained with the ERP analysis collapsed across target type; P300 also decreased in amplitude for increasingly off-diagonal cells. These results were confirmed by the following analysis. Each of the 16 cells of the confusion matrix in Experiment 2 was accorded a "distance" value depending on its position relative to the diagonal. For example, for the $600-\mathrm{Hz}$ target identified as $1,700 \mathrm{~Hz}$, the distance value was 2 ; for correctly identified targets, the distance value was 0 . The Spearman rank-order correlations between distance-from-diagonal and P300 for the four subjects in Experiment 2 were $-.71,-.74$, -.88 , and $-.81(\mathrm{~N}=16, \mathrm{p}<.01$, in each case), indicating that P300 amplitude was dependent on the relative distance between presented and perceived targets in the confusion matrix; P300 amplitude was high for targets likely to be confused with each other and low for targets less likely to be confused with each other.

\section{Single-Trial Analysis}

Single-trial P300 measures in detection and recognition. An amplitude difference in an averaged ERP component can result from changes in the component's latency variability across single trials. A singletrial ERP analysis was carried out to investigate this possibility. Following the procedure outlined in the Method section, estimates of P300 amplitude, latency, and latency variability were obtained. Figure 5 displays the mean values of these measures for the 10 subjects in Experiments 1 and 2. Both the mean latency $[F(3,9)=18.6, p<.01$, for both experiments] and the latency variability of $P 300$ across single trials $[F(3,9)=61.7, p<.001]$ increased across ratings 1 to 4. However, these trial-by-trial latency shifts did not contribute significantly to the $\mathrm{P} 300$ amplitude difference between correctly and incorrectly identified targets. It was confirmed that the amplitude of P300 on single trials was greater for recognized than for unrecognized targets for ratings 1,2 , and 3 [simple effects, $F(1,9)>45.3, p<.001$, for each case] but not for rating 4 .

These results were not dependent on the robustness of the single-trial analysis procedure in detecting a P300 component on single trials. For ratings 1 to 4, the percentages of $\mathrm{P} 300$ peaks detected on single trials (at a $95 \%$ confidence level) were $85 \%, 90 \%$, $86 \%$, and $76 \%$ (recognized target trials) and $88 \%$, $85 \%, 86 \%$, and $76 \%$ (unrecognized target trials), re- 
Table 5

Mean Values (Averaged Over Ratings 1 and 2) of P300 Amplitude (in Microvolts) for the Recognition Confusion Matrix

\begin{tabular}{|c|c|c|c|c|c|c|c|}
\hline \multicolumn{3}{|c|}{ Experiment 1} & \multicolumn{5}{|c|}{ Experiment 2} \\
\hline \multirow[b]{2}{*}{$\mathbf{R}$} & \multicolumn{2}{|c|}{$\mathrm{T}$} & \multirow[b]{2}{*}{$\mathbf{R}$} & \multicolumn{4}{|c|}{$\mathbf{T}$} \\
\hline & 900 & 1400 & & 600 & 1100 & 1700 & 2200 \\
\hline $\begin{array}{r}900 \\
1400\end{array}$ & $\begin{array}{l}7.63 \\
4.66\end{array}$ & $\begin{array}{l}5.17 \\
7.07\end{array}$ & $\begin{array}{r}600 \\
1100 \\
1700 \\
2200\end{array}$ & $\begin{array}{l}7.60 \\
4.84 \\
3.70 \\
2.30\end{array}$ & $\begin{array}{l}4.45 \\
8.15 \\
5.16 \\
2.65\end{array}$ & $\begin{array}{l}2.55 \\
3.80 \\
7.88 \\
3.90\end{array}$ & $\begin{array}{l}3.09 \\
3.97 \\
4.85 \\
7.00\end{array}$ \\
\hline
\end{tabular}

Note-There are 4 cells for the two targets in Experiment 1 and 16 cells for the four targets in Experiment 2. Target $(T)$ and response ( $R$ ) values are given in Hertz.

spectively. These proportions did not differ significantly. Thus, the P300 amplitude difference between recognized and unrecognized targets did not depend on the number of $P 300$ peaks reliably associated with these two stimulus-response categories.

Prediction of behavioral recognition from singletrial P300 amplitude. Since the amplitude of $\mathrm{P} 300$ elicited by target stimuli on single trials was large if the targets were detected and correctly identified, we attempted to predict behavioral recognition from a knowledge of the detection rating and P300 amplitude. We used a binary classification algorithm that maximized the correct classification of targets as correctly or incorrectly recognized, given detection rating and P300 amplitude. The mean classification percentages (across trials and across subjects) for recog-

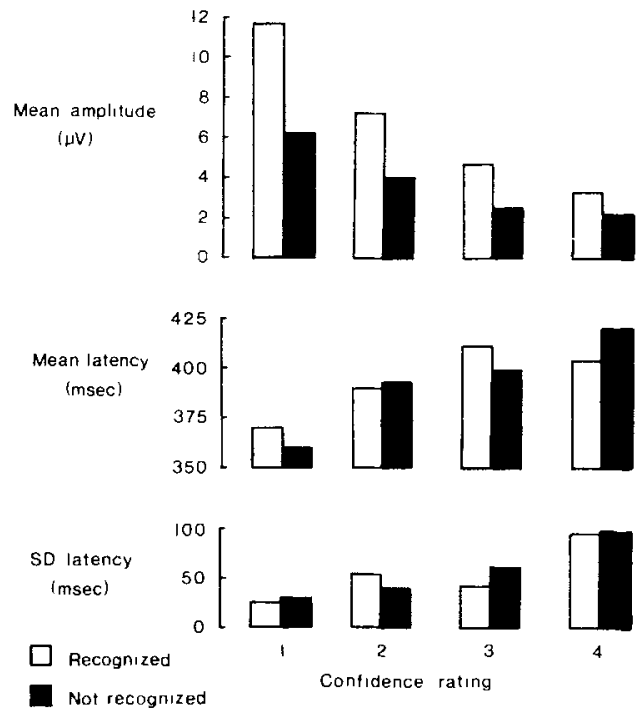

Figure 5. Mean amplitude, latency, and latency variability of P300 (at Cz) across single trials, obtained using an adaptive filtering and peak detection routine. Averaged values across the 10 subjects in Experiments 1 and 2. nized target trials were $90 \%, 77 \%, 68 \%$, and $54 \%$ for ratings 1 to 4 , respectively. The corresponding values for unrecognized target trials were $87 \%, 77 \%$, $64 \%$, and $55 \%$. Thus, behavioral recognition could be predicted at a significant level for ratings 1,2 , and 3. For rating 4 , however, prediction was near chance level.

\section{Principal Components Analysis}

The results obtained with the baseline-to-peak and single-trial measures of $\mathbf{P} 300$ amplitude could be confounded by contributions from components that are adjacent to or overlap the latency range of P300. To account for this possibility, the target ERPs were subjected to a principal components analysis designed to identify independent components contributing to the ERP waveform. Seven factors (components) were extracted by the analysis. The first two of these factors accounted for $88 \%$ of the total variance in the data. The factor loadings for the remaining factors were very low in comparison with those of Factors 1 and 2. The scree test, which probes for significant decreases in the eigenvalues of the covariance matrix as more factors are considered, showed that the proportion of variance accounted for did not change significantly after the second factor. More importantly, only Factors 1 and 2 were significantly affected by the experimental variables, as described below. Thus, only Factors 1 and 2 were retained. Only the results of the PCA for the covariance data matrix are presented, since the results were essentially the same for both the covariance and crossproducts matrices, Factors 1 and 2 being affected similarly by the experimental variables in the two cases.

Figure 6 shows plots of the factor loadings against variables (time points) for Factors 1 and 2. Factor 1 , which accounted for $66.6 \%$ of the total variance, has a form similar to the $\mathrm{P} 300$ component in the average ERP, having a slowly rising and falling waveshape, with an onset latency of about $175 \mathrm{msec}$ and a peak latency of about $360 \mathrm{msec}$. Factor 2, which accounted for $21.8 \%$ of the variance in the data, has a slowly rising sustained positive waveshape, with an onset latency of about $200 \mathrm{msec}$ and a peak latency of about $700 \mathrm{msec}$, and may be related to the "slow wave" reported in previous studies.

Figure 7 plots the mean factor scores for Factors 1 and 2 as a function of the experimental conditions. Each set of scores was subjected to a three-way analysis of variance (correctness of recognition $\times$ detection rating $x$ electrode site).

For Factor 1, there were significant main effects of both correctness of recognition $[F(1,9)=81.1, p<$ $.001]$ and detection rating $[\mathrm{F}(3,27)=121.9, \mathrm{p}<.001]$. The interaction between these factors was also significant $[F(3,27)=53.0, p<.001]$. This pattern of re- 

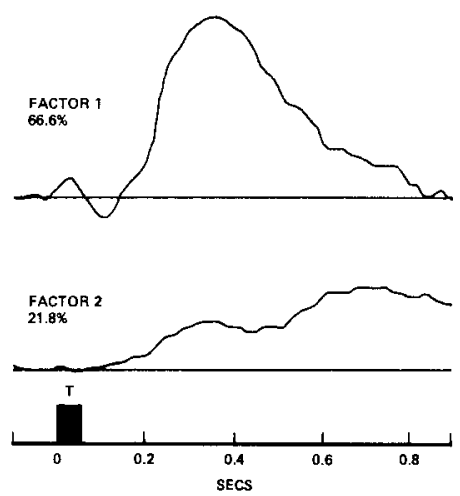

Figure 6. Basis waveforms (factor loadings plotted against time) for the two principal factors extracted by the PCA. The time of presentation of the target and the proportion of variance accounted for by ench factor are also shown.

sults is the same as that obtained with the baselinepeak and single-trial P300 measures. As Figure 7 indicates, Factor 1 was influenced by both detection and recognition in the same way as $\mathrm{P} 300$ amplitude was. Factor 1 scores decreased across ratings 1 to 4 , and they were higher for recognized than for unrecognized targets, but only for detection ratings 1,2 , and 3 [simple effects, $F(1,9)>30.7, p<.001$, for each case] and not for rating 4. A significant effect of electrode site was also found $[F(2,18)=26.6, p<.001]$. As Figure 7 shows, and in confirmation of the P300 results, Factor 1 was smaller at $\mathrm{Fz}$ than at $\mathrm{Cz}$ and $\mathrm{Pz}$.

For Factor 2, there was a significant effect of correctness of recognition only $[F(1,9)=10.8, p<.01]$; detection rating, however, had no significant effect on Factor $2[F(3,27)<1]$. As Figure 7 also indicates, Factor 2 was larger for recognized targets than for
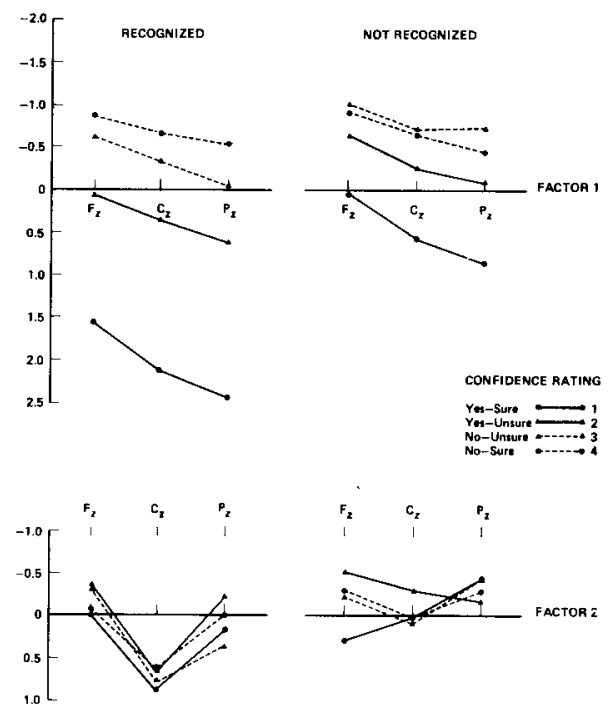

Figure 7. Mean factor scores for the two principal factors extracted by the PCA for correctly and incorrectly recognized targets at each confidence rating of detection and each electrode site. unrecognized targets, but there was no difference in the size of this factor across detection ratings 1 to 4 . The interaction between detection rating and correctness of recognition was also nonsignificant $[F(3,27)$ $<1]$. The effect of electrode site on Factor 2 was only marginally significant $[F(2,18)=4.13, p<.05$; but $\mathrm{p}<.10$, using a conservative degrees of freedom of $(1,9)$ for a repeated measures design]. A significant interaction between electrode site and recognition was also found $[F(2,18)=6.1, p<.01]$, indicating that Factor 2 was more positive for recognized targets than for unrecognized targets at both $\mathrm{Cz}$ [simple effects, $F(1,9)=13.9, \mathrm{p}<.005$ ] and $\mathrm{Pz}$ [simple effects, $F(1,9)=6.9, p<.05]$ but that the factor scores did not differ at Fz.

\section{DISCUSSION}

The results corroborate and extend those presented in our previous brief report on event-related potentials in detection and identification (Parasuraman \& Beatty, 1980). A target stimulus correctly perceived with respect to both its presence and its identity is associated with changes in the $\mathrm{N} 100$ and P300 brain potentials that disclose temporal aspects of the processing that the stimulus undergoes in the nervous system. As hypothesized, however, a slow positive shift (SPS) potential was found to contribute to changes in the baseline to peak amplitude of P300 in the averaged ERP. A number of studies have reported on the contribution of slow potential shifts to the P300 "complex"; Ruchkin et al. (1980), for example, have recently reported on interactions between the slow wave, CNV resolution and the P300 in a signal detection task. In the present study, an SPS having a similar waveshape but different scalp topography to the slow wave was active in a latency range that overlapped with that of the $P 300$ component. When the relative contributions of these two components were separated by multivariate analysis, however, our previous finding that $\mathrm{P} 300$ amplitude varies with both detection and recognition was still obtained.

Three measures of $\mathrm{P} 300$, then, support the relation of P300 to both detection and recognition: (1) baselineto-peak amplitude in the averaged ERP; (2) baselineto-peak amplitude of P300 in single-trial ERPs; and (3) factor scores on a "P300" component extracted by principal components analysis. An unexpected finding from this analysis was that, while SPS amplitude was greater for correctly identified stimuli than for unrecognized stimuli (at central and parietal scalp sites), the SPS did not vary with detection. Thus, a stimulus that must be both detected and recognized elicits a complex of temporally overlapping brain potentials, N100, P300, and SPS, each of which is differentially related to the processing of the stimulus: an early negative potential (N100) that varies in am- 
plitude only with processing related to the detection of the stimulus; a later positive potential (P300) that varies with both detection and recognition; and a final slow positive potential that is unaffected by detection and varies only with recognition. In addition to these ERP amplitude changes, variations in ERP latency were associated only with detection rating. The latencies of both $\mathrm{N} 100$ and $\mathrm{P} 300$ increased across detection ratings 1 to 4 , and the variability of $P 300$ latency across single trials also increased as subjects' confidence that a target was presented decreased. Similar results were reported by Kerkhof (1978) and K. C. Squires et al. (1973) for simple detection tasks. However, recognized and unrecognized targets did not differ in either N100 or P300 latency.

These results support the view that detection and recognition are partially independent, concurrent processes in perception, but that the process of detection begins before the process of identification. Depending on factors such as target detectability, a priori target probabilities, and instructions regarding the speed-accuracy tradeoff, either process may terminate before or after the other. According to this view, identification does not necessarily have to follow detection or detection follow identification. Nolte (1967) has shown how an ideal observer would optimize performance in a combined detection and recognition task by storing updated probability estimates of each target as the stimulus is processed; these estimates represent the information or accumulating evidence on which either detection or recognition responses can be based. Thus, detection and recognition can be considered to proceed together as the nervous system gathers probabilistic information about the stimulus. Swets et al. (1978) have provided further support for this view in a study of the perception of visual sonarlike signals in a spectrographic display exposed progressively more completely over successive observation intervals. They found that detection and recognition accuracy increased together over observation intervals in a manner consistent with Nolte's (1967) model. Recently, Green and Birdsall (1978) have also shown that the analysis of recognition performance can be treated in the same way as detection performance and, furthermore, that recognition can be predicted, to a degree, from detection performance using the theorem of Starr et al. (1975). Although the predicted and obtained recognition probabilities were ordered monotonically, the theorem overestimated the performance of the observers in this study. In contrast, a combined behavioral and physiological method of prediction of recognition on individual trials based on detection rating and the amplitude of P300 on single trials fared quite favorably.

These results indicate that detection and recognition responses are based on the same underlying buildup of neural information regarding an uncertain stimulus and hence that detection and recognition are related processes. Nevertheless, the finding that detection and recognition were differentially associated with three independent ERP components suggests that these temporally overlapping processes are partially independent. This view also suggests certain relations between detection and recognition performance. Whereas, on the one hand, a stimulus that cannot be identified can often be accurately detected, in some instances, on the other hand, a stimulus that is not detected may nevertheless be correctly identified; in short, either detection without recognition or recognition without detection is a tenable outcome. This proposition follows directly from the basic tenets of signal detection theory and other continuous models of detection (Green \& Swets, 1966). Threshold models of detection, however, in particular, high-threshold theory (Blackwell, 1963) and two-state low-threshold theory (Luce, 1963b), predict that recognition responses emitted in "nondetect" states should be distributed randomly among stimuli, and thus that recognition performance should be no better than chance when subjects report that no stimulus was presented (Lindner, 1968; Rollman \& Nachmias, 1972). Our results are clearly to the contrary. In both Experiments 1 and 2, subjects identified presented targets even when they were fairly confident that no target had been presented (rating 3). These responses were correct significantly more often than chance in 9 of the 10 subjects. Even when very confident targetabsent reports were made (rating 4), recognition performance was above chance in 6 of the 10 subjects. Thus, even though the underlying neural information on which detection and recognition responses are based may be insufficient to exceed a criterion for detection, it may nevertheless be sufficient for better than chance recognition. The finding that $\mathrm{P} 300 \mathrm{am}-$ plitude was greater for correctly recognized targets than for unrecognized targets, even for doubtful target-absent reports, fits well with this view. P300 amplitude indexed whether a particular target would be recognized at above-chance levels even when the subject reported that the target was not present.

The differential behavior of N100 and P300 in the present study with respect to detection and recognition parallels the differential involvement of these brain potential components with stimulus selection and target detection in selective information processing (Hillyard et al., 1973; Hillyard \& Picton, 1979). Although a slow negative shift in potential that overlaps with $\mathrm{N} 100$ has been reported in selective attention studies (Näätänen \& Michie, 1979; Parasuraman, 1980), there are no data on the possible existence of such a negative shift in signal detection situations. Our data do not provide a complete answer to this question, but no such component was obtained in the PCA. 
Although variations in the likelihood ratio criterion for detection (induced by the use of a rating scale) affect N100 and P300 similarly, our results support K. C. Squires et al.'s (1973) view that these components index quite different processes. K. C. Squires et al. found that N100 varied in a fashion similar to the changes produced by varying signal intensity in a "passive" listening situation, but that P300 was unrelated to any passively evoked brain potential. They suggested therefore that variations in N100 reflected changes in subjective signal intensity (or the quantity of information received by the signal detecting system), whereas P300 was associated with the subsequent decision processes based on the utilization of this information. Our findings indicate that P300 amplitude may vary as a function of the quality of probabilistic neural information relative to an internal criterion; changes in the criterion for either detection or recognition responses are reflected in P300 amplitude. The variation of P300 amplitude within the recognition confusion matrix provides further support for this view. P300 amplitude varied as a decreasing function of the similarity between perceived and presented stimuli.

Both effects obtained on P300 can also be interpreted to mean that $\mathrm{P} 300$ is associated with postdecisional evaluation processes-specifically, that P300 amplitude is larger the less the equivocation (Ruchkin \& Sutton, Note 2) or the greater the uncertainty resolution is (Sutton, Braren, Zubin, \& John, 1965). However, since the SPS, unlike P300, varied only with recognition and since it developed only after the resolution of the P300, the SPS would also have to be called postdecisional for $\mathrm{P} 300$ to be postdecisional. The evaluation process underlying the SPS would also have to be specific to the categorical information contained in the stimulus. Ruchkin et al.'s (1980) view is that P300 represents an intermediate stage of event evaluation common to different judgment tasks and that the slow wave reflects an ensuing final evaluation that differs according to the complexity of the judgment.

One difficulty in relating our results to those of Ruchkin et al. (1980), however, or to other previous studies reporting on slow positive waves is that the SPS obtained in this study had a different scalp distribution to that usually reported for the slow wave. The previously described slow wave has generally been characterized as negative frontally, increasingly positive with more posterior derivations, and with a parietal maximum (McCarthy \& Donchin, 1976; K. C. Squires, Donchin, Herning, \& McCarthy, 1977; N. K. Squires, Donchin, \& K. C. Squires, 1977). N. K. Squires et al., 1975). Although such a scalp topography for SPS was seen in the ERP waveforms of some of our subjects, the SPS factor extracted by PCA was a positive potential that was maximal centrally. Furthermore, the slow wave and $\mathrm{P} 300$ have usually been found to be influenced similarly by the same experimental variables. Recently, however, experimental dissociation of the slow wave and P300 has been reported by Roth, Ford, and Kopell (1978) and by Ruchkin et al. (1980). The question to be resolved, thus, is whether the SPS obtained in the present study is a component similar to the slow wave that varies in topography with different experimental manipulations or is a different component.

The "identity" of the SPS notwithstanding, the temporal relation of this brain component to the earlier N100 and P300 components supports the proposed parallel stage view of detection and recognition. Assuming that the processes manifested in these brain potentials are indeed active at different times (as suggested by their onset and peak latencies), we conclude that, whereas stimulus processing related to detection begins before that associated with recognition, at later stages detection and recognition are concurrent processes.

\section{REFERENCE NOTES}

1. Donchin, E., \& Heffley, E. Multivariate analysis of eventrelated potential data: A tutorial review. In D. A. Otto (Ed.), Multidisciplinary perspectives in event-related brain potential research (EPA-600/9-77-043). Washington, D.C: U.S. Environmental Protection Agency, 1978.

2. Ruchkin, D. S., \& Sutton, S. Equivocation and P300 amplitude. In D. A. Otto (Ed.), Multidisciplinary perspectives in eventrelated brain potential research (EPA-600/9-77-043). Washington, D.C: U.S. Environmental Protection Agency, 1978.

\section{REFERENCES}

Barhett, G., Halliday, A. M., Halliday, E., \& Rudolf, N. The later components of the somatosensory evoked response and the P300 in a threshold detection task. In J. E. Desmedt (Ed.), Cognitive components in cerebral event-related brain potentials and selective attention. Basel: Karger, 1979.

BLACKWELL, H. R. Neural theories of simple visual discriminations. Journal of the Optical Society of America, 1963, 53, 129-160.

Desmedt, J. E., \& DeBecken, J. Wave form and neural mechanism of the decision P350 elicited without pre-stimulus CNV or readiness potential in random sequences of near-threshold auditory clicks and finger stimuli. Electroencephalography and Clinical Neurophysiology, 1979, 47, 648-670. (a)

Desmedt, J. E., \& Debecker, J. Slow potential shifts and decision P350 interactions in task with random sequences of near-threshold clicks and finger stimuli delivered at regular intervals. Electroencephalography and Clinical Neurophysiology, 1979, 47, 671-679. (b)

Dixon, W. J. Biomedical computer programs. P-series. Los Angeles: University of California Press, 1977.

Donchin, E., \& Smiтh, D. B. D. The contingent negative variation and the late positive wave of the average evoked potential. Electroencephalography and Clinical Neurophysiology, 1970, 29, 201-203.

Green, D. M., \& Birdsall, T. G. Detection and recognition. Psychological Review, 1978, 85, 192-206.

Green, D. M., McKey, M. J., \& Licklider, J. C. R. Detection of a pulsed sinusoid in noise as a function of frequency. Journal of the Acoustical Society of America, 1959, 31, 1446-1452. 
Green, D. M., \& Swets, J. A. Signal detection theory and psychophysics. New York: Wiley, 1966.

Grey, D. R., \& Morgan, B. J. T. Some aspects of ROC curve fitting: Normal and logistic models. British Journal of Mathematical Psychology, 1972, 9, 128-139.

Hillyard, S. A., Hink, R. F., Schwent, V. L., \& Picton, T. W. Electric signs of selective attention in the human brain. Science, 1973, 182, 177-180.

Hilly ARD, S. A., \& Picton, T. W. Event-related brain potentials and selective information processing in man. In J. Desmedt (Ed.), Cognitive components in cerebral event-related brain potentials and selective attention. Basel: Karger, 1979.

Hillyard, S. A., Squires, K. C., Bauer, J. W., \& Lindsay, P. H. Evoked potential correlates of signal detection. Science, $1971,172,1357-1360$.

Ke RKhoF, G. A. Decision latency: The $P 3$ component in auditory signal detection. Neuroscience Letters, 1978, 8, 289-294.

LiNDNER, W. A. Recognition performance as a function of detection criterion in a simultaneous detection-recognition task. Journal of the Acoustical Society of America, 1968, 44, 204-211.

LuCE, R. D. Detection and recognition. In R. D. Luce, R. R. Bush, \& E. Galanter (Eds.), Handbook of mathematical psychology. New York: Wiley, 1963. (a)

Luce, R. D. A threshold theory for simple detection experiments. Psychological Review, 1963, 70, 61-79. (b)

Luce, R. D., \& Green, D. M. Detection, discrimination and recognition. In E. C. Carterette \& M. P. Friedman (Eds.), Handbook of perception (Vol. 2): Psychophysical judgement and measurement. New York: Academic Press, 1974.

McCallum, W. C., \& KnotT, J. R. (Eds.). Event-related slow potentials of the brain. Electroencephalography and Clinical Neurophysiology Supplement, 1973, 33.

McCarthy, G., \& Donchin, E. The effects of temporal and event uncertainty in determining the waveforms of the auditory event-related potential (ERP). Psychophysiology, 1976, 13, 581-590.

McGillem, C. D., \& Aunon, J. I. Measurements of signal components in single visually evoked brain potentials. IEEE Transactions on Biomedical Engineering, 1977, BME-24, 232-241.

NÄ̈̈ÄNEN, R., \& MichiE, P. Early selective-attention effects on the evoked potential: $A$ critical review and reinterpretation. Biological Psychology, 1979, 8, 81-136.

Nolte, L. W. Theory of signal detectability: Adaptive optimum receiver design. Journal of the Acoustical Society of America, $1967,42,773-777$.

Parasuraman, R. Effects of information processing demands on slow negative shift latencies and N100 amplitude in selective and divided attention. Biological Psychology, 1980, 11, 217-233.

Parasuraman, R., \& BeatTy, J. Brain events underlying the detection and recognition of weak sensory signals. Science, $1980,210,80-83$.

Paul, D. D., \& Sutron, S. Evoked potential correlates of response criteria in auditory signal detection. Science, 1972, 177, 362-364.

Rollman, G. B., \& Nachmias, J. Simultaneous detection and recognition of chromatic flashes. Perception \& Psychophysics, 1972, 12, 309-314.
Roth, W. T., Ford, J. M., \& KopelL, B. S. Long-latency evoked potentials and reaction time. Psychophysiology, 1978, 15, 17-23.

Ruchkin, D. S., Sutron, S., \& STEgA, M. Emitted P300 and slow wave event-related potentials in guessing and detection tasks. Electroencephalography and Clinical Neurophysiology, $1980,49,1-14$.

Simpson, A. J., \& FitTen, M. J. What is the best index of detectability? Psychological Bulletin, 1973, 80, 481-488.

Simson, R., Vaughan, H. G., \& RitTer, W. The scalp topography of potentials in auditory and visual go and no-go tasks. Electroencephalography and Clinical Neurophysiology, 1977, 43, 864-875.

Sinyer, E., Hillyard, S. A., \& Galambos, R. Similarities and differences among the $P 3$ waves to detected signals in three modalities. Psychophysiology, 1980, 17, 112-122.

Squires, K. C., Donchin, E., Herning, R. I., \& McCarthy, G. On the influence of task relevance and stimulus probability on event-related-potential components. Electroencephalography and Clinical Neurophysiology, 1977, 42, 1-14.

Squires, K. C., Hillyard, S. A., \& Lindsay, P. H. Vertex potentials evoked during auditory signal detection: Relation to decision criteria. Perception \& Psychophysics, 1973, 14, 265-272.

Squires, K. C., Squires, N. K., \& Hillyard, S. A. Vertex evoked potentials in a rating-scale task detection task: Relation to signal probability. Behavioral Biology, 1975, 13, 21-34. (a)

Squires, K. C., Squires, N. K., \& Hillyard, S. A. Decisionrelated cortical evoked potentials during an auditory signal detection task with cued observation intervals. Journal of Experimental Psychology: Human Perception and Performance, 1975, 1, 268-279. (b)

Squires, N. K., Donchin, E., \& Squires, K. C. Bisensory stimulation: Inferring decision-related processes from the P300 component. Journal of Experimental Psychology: Human Perception and Performance, 1977, 3, 299-315.

Squires, N. K., Squires, K. C., \& Hillyard, S. A. Two varieties of long-latency positive waves evoked by unpredictable auditory stimuli in man. Electroencephalography and Clinical Neurophysiology, 1975, 38, 387-401.

Starr, S. J., Metz, C. E., Lusted, L. B., \& Goodenough, D. J. Visual detection and localization of radiographic images. Radiology, 1975, 116, 533-538.

Sutton, S., Braren, M., Zubin, J., \& John, E. R. Evoked potential correlates of stimulus uncertainty. Science, 1965, 150, 1187-1188.

Swets, J. A., \& Birdsall, T. G. Repeated observation of an uncertain signal. Perception \& Psychophysics, 1978, 23, 269-274.

Swets, J. A., Green, D. M., Getty, D. J., \& Swets, J. A. Signal detection and identification at successive stages of observation. Perception \& Psychophysics, 1978, 23, 275-289.

TANnen, W. P. A theory of recognition. Journal of the Acoustical Society of America, 1956, 28, 882-888.

(Manuscript received August 3, 1981; revision accepted for publication September 23, 1981.) 